\title{
COMPARAÇÃO BIOMECÂNICA DA CORRIDA ENTRE INDIVÍDUOS COM DIFERENTES NÍVEIS DE FORÇA DE MEMBROS INFERIORES
}

\author{
RUNNING BIOMECHANICAL COMPARISON BETWEEN INDIVIDUALS \\ WITH DIFFERENT LEVELS OF LOWER LIMBS STRENGTH
}

\section{COMPARACIÓN BIOMECÁNICA DE LA CARRERA ENTRE INDIVIDUOS COM DIFERENTES NIVELES DE FUERZA DE MIEMBROS INFERIORES}

\author{
Wilson Pereira Lima \\ https://orcid.org/0000-0002-1832-5108 (iD \\ http://lattes.cnpq.br/9144131870576428 \\ Universidade de São Paulo (São Paulo, SP - Brasil) \\ wplima22@gmail.com \\ Carlos Alberto Cardoso Filho \\ https://orcid.org/0000-0003-3204-9397 (iD) \\ http://lattes.cnpq.br/0248219632673942 \\ Universidade de São Paulo (São Paulo, SP - Brasil) \\ carlos.filho@alumni.usp.br \\ Maura de Arruda Botelho Colturato \\ https://orcid.org/0000-0003-3649-5060 (iD \\ http://lattes.cnpq.br/3635399924473627 5 \\ Universidade de São Paulo (São Paulo, SP - Brasil) \\ mauracolturato@gmail.com \\ João Pedro dos Santos Ferreira Moreira Pinho \\ https://orcid.org/0000-0003-4763-9410 ic \\ http://lattes.cnpq.br/1494809217440737 \\ Universidade de São Paulo (São Paulo, SP - Brasil) \\ pinhojp@usp.br
}

Ana Paula da Silva Azevedo

https://orcid.org/0000-0003-4866-145X (iD

http://lattes.cnpq.br/7492408229689062 9

Universidade de São Paulo (São Paulo, SP - Brasil)

anaazevedo@usp.br

Júlio Cerca Serrão

https://orcid.org/0000-0002-3646-3387 (D)

http://lattes.cnpq.br/9124685212860479

Universidade de São Paulo (São Paulo, SP - Brasil)

jcserrao@usp.br

\section{Resumo}

A corrida é uma prática largamente utilizada por aqueles que almejam manter ou aprimorar a aptidão física. O treinamento de força tem sido recomendado como forma de aumentar o desempenho e reduzir o risco de lesão em praticantes de corrida, no entanto, a relação entre força muscular e mecânica da corrida ainda necessita de 
melhor compreensão. O objetivo do presente estudo foi comparar a biomecânica da corrida entre indivíduos praticantes de musculação, com diferentes níveis de força absoluta no agachamento. Inicialmente participaram do estudo 24 voluntários. Baseado na força absoluta em 10RM no agachamento, os voluntários foram divididos em tercis: grupo de maior força (GMaF), grupo de menor força (GMeF) e grupo intermediário. Para comparação da mecânica da corrida foram analisados o GMaF e GMeF. Foram coletados parâmetros cinéticos e cinemáticos durante corrida em esteira. Não se observou diferenças entre os grupos para nenhuma das variáveis investigadas.

Palavras-chave: Mecânica da Corrida; Força Muscular; Locomoção.

\section{Abstract}

Running is a practice widely used by those who aim to maintain or improve their physical fitness. Strength training has been recommended to increase performance and reduce injury risk in runners, however, the relationship between muscle strength and running mechanics still requires a better understanding. The aim of the present study was to compare the running biomechanics among resistance-trained men with different levels of absolute strength in the back squat. Initially, 24 volunteers participated in the study. Based on the 10RM absolute strength in the back squat, the volunteers were divided into tertiles: highest strength group (GMaF), lowest strength group (GMeF) and intermediate group. For comparison of running mechanics, GMaF and GMeF were analyzed. Kinetic and kinematic parameters were collected during treadmill running. There were no differences between groups for any of the investigated variables.

Keywords: Running Mechanics; Muscle Strength; Locomotion.

\section{Resumen}

Correr es una práctica muy utilizada por quienes tienen como objetivo mantener o mejorar su forma física. El entrenamiento de fuerza se ha recomendado como una forma de aumentar el rendimiento y reducir el riesgo de lesiones en los corredores, sin embargo, la relación entre la fuerza muscular y la mecánica de carrera aún requiere una mejor comprensión. El objetivo del presente estudio fue comparar la biomecánica de la carrera entre culturistas, con diferentes niveles de fuerza absoluta en la sentadilla. Inicialmente, participaron en el estudio 24 voluntarios. Con base en la fuerza absoluta de 10RM en la sentadilla, los voluntarios se dividieron en terciles: grupo de mayor fuerza (GMaF), grupo de menor fuerza (GMeF) y grupo intermedio. Para comparar la mecánica de la carrera, se obtuvieron GMaF y GMeF. Los parámetros cinéticos y cinemáticos se recopilaron durante la carrera en cinta. No hubo diferencias entre los grupos para ninguna de las variables investigadas.

Palabras clave: Mecánica de Carrera; Fuerza Muscular; Locomoción.

\section{INTRODUÇÃO}

A corrida é uma das atividades físicas mais populares entre aqueles que desejam se manter bem fisicamente; havendo forte evidência de que melhores níveis de aptidão física promovem maior longevidade (FIELDS et al., 2010). Além disso, por ser uma atividade acessível e de baixo custo, é uma opção atrativa para muitas pessoas (JOHNSTON et al., 2003).

Por outro lado, concomitantemente a esta popularidade, há evidências de que a prática da corrida está associada ao aumento do risco de lesões (DAVIS; BOWSER; MULLINEAUX, 2016; LIEBERMAN, 2012; VAN DER WORP et al., 2015), com incidência que pode variar entre 19,4\% até 79,3\% (VAN GENT et al., 2007). Especificamente no Brasil, a metanálise conduzida por Borel e colaboradores (2019) indicou prevalência de lesão de 36,5\% em corredores recreacionais. Dentre os principais fatores relacionados com lesões estão, o volume e a frequência de treinamento de corrida (BLAGROVE et al., 2020; BOREL et al., 2019), piso irregular, calçado inapropriado, falta de flexibilidade, desalinhamento de membros inferiores 
(JOHNSTON et al., 2003) e fraqueza muscular (BECKER; NAKAJIMA; WU, 2018; FIELDS et al., 2010; JOHNSTON et al., 2003; MUCHA et al., 2017). De maneira que estratégias para prevenção de lesão em praticantes de corrida, em geral, é um assunto importante para profissionais envolvidos com a prescrição e orientação desta atividade.

Embora seja difícil de prever lesões na corrida, haja vista a combinação de diversos fatores que podem ser associados com lesões, e que alguns corredores se lesionam e outros não, parece haver um consenso sobre a recomendação do fortalecimento dos músculos de membros inferiores como uma forma de reduzir a probabilidade de lesão na corrida (BECKER; NAKAJIMA; WU, 2018; JOHNSTON et al., 2003; MOFFIT et al., 2020). Em tese, exercícios destinados ao aprimoramento da força muscular aumentam o stiffness dos tecidos conectivos e consequentemente aumentam a sua tolerância para maiores cargas aplicadas ao aparelho locomotor (BOHM; MERSMANN; ARAMPATZIS, 2015).

Ademais, por além de ser considerado uma estratégia cabível para diminuir a probabilidade de lesões, o treinamento de força também se mostra importante para melhoria da economia de corrida e desempenho em corridas de média e longa distâncias (ALCARAZIBAÑEZ; RODRÍGUEZ-PÉREZ, 2018; BALSALOBRE-FERNÁNDEZ; SANTOS-CONCEJERO; GRIVAS, 2016; LI et al., 2019; MIKKOLA et al., 2011; YAMAMOTO et al., 2008). Ainda, a literatura aponta forte relação entre a capacidade de produzir força de membros inferiores, por meio do exercício agachamento, com o desempenho em corridas de curta distância (COMFORT et al., 2014; COMFORT; BULLOCK; PEARSON, 2012; WISLØFF et al., 2004).

Embora o treinamento de força seja utilizado como uma estratégia tanto para prevenção de lesão, como para aumentar o desempenho de praticantes de corrida, a relação entre força máxima e a biomecânica da corrida ainda não são claras na literatura. Além disso, a maioria dos estudos que analisaram a biomecânica da corrida, não tiveram como propósito investigar indivíduos que participam de programas típicos de academias, como por exemplo, praticantes de musculação que usualmente utilizam a corrida em esteira como parte de seu programa de condicionamento físico. Neste sentido, o objetivo do presente estudo foi comparar a biomecânica da corrida entre indivíduos praticantes de musculação, com diferentes níveis de força máxima absoluta, no exercício agachamento livre. 


\section{MÉTODO}

\section{Abordagem Experimental do Problema}

Os voluntários foram avaliados em pelo menos três sessões, sendo pelo menos duas sessões prévias para determinação da velocidade de corrida em esteira instrumentada e da carga do exercício agachamento livre; e uma sessão experimental para obtenção dos parâmetros biomecânicos da corrida em esteira.

\section{Amostra}

Inicialmente, nas sessões prévias, participaram deste estudo 24 voluntários do sexo masculino (Idade: 29,2 \pm 5,0 anos; Altura: 1,76 \pm 0,04 m; Massa corporal: 86,7 \pm 11,1 Kg); praticantes de musculação, com frequência semanal de treino de 4,8 $\pm 0,9$ dias, sendo 1,9 \pm 0,5 dias de treino para membros inferiores; e que utilizam a corrida em esteira como parte de seu programa de treinamento, com frequência semanal de 2,5 $\pm 1,2$ dias e duração de 21,7 \pm 9,5 min por sessão de corrida. Com base na carga absoluta mobilizada no exercício agachamento livre, em 10RM, os voluntários foram divididos em tercis, sendo um grupo de maior força (GMaF), um grupo de menor força (GMeF) e um grupo intermediário. Na sessão experimental somente foram avaliados, para efeito de comparação, os grupos GMaF e GMeF $(n=8$; Idade: $29,3 \pm 5,5$ anos e 29,3 $\pm 5,9$ anos; Altura: $1,77 \pm 0,04$ m e 1,73 $\pm 0,04$ m; Massa corporal: $94,9 \pm 6,1 \mathrm{Kg}$ e 78,3 $\pm 11,7 \mathrm{Kg}$ respectivamente).

Para inclusão dos indivíduos na amostra foram adotados os seguintes critérios: sexo masculino; ter entre 18 e 40 anos de idade; possuir experiência com o treinamento de força por um período superior a um ano; executar semanalmente o exercício agachamento livre na rotina de treinamento por pelo menos um ano; utilizar a corrida em esteira como parte do programa de condicionamento físico por pelo menos um ano; não apresentar alguma desordem neuromuscular, cardiovascular, ou qualquer outra patologia que limitasse a realização do protocolo.

Os voluntários foram informados sobre os objetivos, procedimentos, riscos e benefícios da investigação e assinaram Termo de Consentimento Livre e Esclarecido (TCLE). Todos os procedimentos da pesquisa foram aprovados por Comitê de Ética em Pesquisa da Universidade de São Paulo (CAAE 13315519.3.0000.5391). 


\section{PROCEDIMENTOS}

\section{Determinação da velocidade de corrida}

Nas sessões para procedimentos prévios, com intervalo mínimo de 48 horas entre as sessões, foi realizado o teste de corrida em esteira, onde foi determinada a velocidade autosselecionada utilizada na sessão de coleta de dados e realizado o procedimento para determinação da carga do exercício agachamento livre.

Inicialmente era mensurada a estatura e massa corporal do voluntário. Em seguida foi determinada a velocidade de corrida autoselecionada. Os voluntários primeiramente realizavam uma familiarização em esteira por um período de 3 minutos, na velocidade inicial de $6 \mathrm{~km} / \mathrm{h}$, com incrementos de velocidade a critério do voluntário. Além disso, eram devidamente instruídos sobre a escala de percepção subjetiva de esforço (PSE), adaptada de (FOSTER et al., 2001).

Após a familiarização, os voluntários foram orientados a alcançar uma velocidade na qual pudessem correr em intensidade moderada por 20 minutos (ADAMS et al., 2016; LAVCANSKA; TAYLOR; SCHACHE, 2005; NAWOCZENSKI; LUDEWIG, 1999; QUEEN; GROSS; LIU, 2006). Os ajustes de velocidade eram realizados sem que os voluntários tivessem acesso a velocidade da esteira. A esteira era inicializada na velocidade de $6 \mathrm{~km} / \mathrm{h}$ e de acordo com a instrução do próprio sujeito a velocidade era gradualmente aumentada. Quando o voluntário relatava ter atingido velocidade que the permitia correr em intensidade moderada por 20 minutos, a velocidade era mantida por 1 minuto. Após este período, quando a velocidade era confirmada pelo voluntário, ela era considerada estabilizada. Do contrário, novos ajustes de velocidade eram permitidos e ela era novamente mantida por 1 minuto; este procedimento era repetido até se atingir a estabilização da velocidade. Ao final do teste de corrida, o voluntário indicava a PSE como forma de familiarização com a escala.

O tempo médio gasto pelos voluntários do GMaF e GMeF foi de 02:18 \pm 00:22 min e 03:12 \pm 01:14 min na primeira sessão prévia e 02:07 \pm 00:17 min e 02:25 \pm 00:26 min na segunda sessão prévia; as velocidades obtidas foram 8,7 $\pm 1,2 \mathrm{~km} / \mathrm{h}$ (PSE 2,5 \pm 0,5) e 9,8 $\pm 1,6$ km/h (PSE 2,7 \pm 0,6) na primeira sessão prévia; e 8,4 $\pm 1,0$ km/h (PSE 2,6 $\pm 0,7$ ) e 9,2 $\pm 1,4$ km/h (PSE 2,5 $\pm 0,8)$ na segunda sessão prévia respectivamente. Desta forma, a velocidade de corrida 
utilizada na sessão de coletas de dados foi a média das velocidades obtidas em cada sessão para procedimentos prévios, para cada voluntário.

\section{Determinação da carga para o exercício agachamento livre.}

Ainda nas sessões para procedimentos prévios, após o teste de corrida, foi realizado ajuste da carga do exercício agachamento livre (10RM). Foram realizadas 3 séries, com intervalo de 3 minutos entre as séries. A velocidade de movimento não foi controlada, mantendo assim a característica de execução individual dos voluntários. O exercício foi realizado até a falha concêntrica, ou interrompido quando o voluntário reportasse incapacidade de manter a execução de movimento de acordo com os critérios estabelecidos. Um profissional experiente, previamente treinado, observou e orientou os voluntários ao longo do teste com o propósito de assegurar os critérios de execução de movimento determinados para o exercício.

O Agachamento foi realizado com pesos livres. Os voluntários deveriam apoiar a barra na região posterior dos ombros, posicionar os pés da maneira mais confortável e com afastamento equivalente à largura dos ombros. Ao longo da execução do movimento eles deveriam manter a coluna na posição mais estável possível. Na fase descendente do movimento, os joelhos deveriam flexionar até que o ângulo entre a coxa e a tíbia atingisse $90^{\circ}$. Foi considerado como um movimento completo quando na fase ascendente os voluntários estendiam completamente os joelhos e quadril.

A primeira sessão para ajustar a carga do agachamento livre foi iniciada com um aquecimento específico efetivado por meio de 10 repetições com aproximadamente $50 \%$ da carga de 10RM previamente reportada pelo voluntário. Após aquecimento, a primeira série foi realizada com a carga previamente reportada pelo voluntário. Quando o voluntário realizava um número de repetições menor ou maior do que 10, a carga era ajustada para a próxima série. A carga da terceira série foi anotada com a finalidade de ser replicada na próxima sessão de ajuste da carga.

A segunda sessão para ajustar a carga do agachamento livre foi iniciada com um aquecimento específico efetivado de forma idêntica ao utilizado na sessão anterior. Após aquecimento, a primeira série foi realizada de acordo com a carga determinada na primeira sessão. Quando o voluntário mantinha 10RM para a carga previamente determinada, ela era considerada como ajustada; do contrário, mais uma sessão prévia de determinação da carga 
era realizada. Nenhum dos voluntários do estudo precisou realizar uma terceira sessão de estabilização da carga ou da velocidade de corrida. A carga média obtida durante o procedimento de ajuste para o agachamento livre foi de $152,5 \pm 18,3 \mathrm{Kg}$ para o GMaF e 101,3 $\pm 8,3 \mathrm{Kg}$ para o GMeF $(\mathrm{p}<0,001)$.

\section{Coleta de dados}

Após um intervalo mínimo de 48 horas da última sessão de procedimentos prévios, foi realizada a sessão experimental.

Para registro da força de reação do solo (FRS), a corrida foi realizada na esteira instrumentada Gaitway HP Cosmos. A FRS vertical foi registrada à $250 \mathrm{~Hz}$. Os parâmetros calculados a partir da curva força-tempo foram: $1^{\circ}$ pico de força, tempo para o $1^{\circ}$ pico de força, taxa de desenvolvimento de força e impulso total.

Para cinemática foram utilizadas 8 câmeras óptico-eletrônicas (Flex 3) com resolução de 0,3 Mpixels e frequência de amostragem de 100 fps (OptiTrack; NaturalPoint, EUA). Marcadores reflexivos (14 $\mathrm{mm} \varnothing$ ) foram colocados nos membros inferiores direito e esquerdo, nas seguintes posições anatômicas: espinha ilíaca ântero-superior; espinha ilíaca póstero-superior; epicôndilo lateral do fêmur; maléolo lateral da fíbula; calcâneo; e segundo metatarso, além de clusters localizados: lateralmente sobre a coxa, na linha formada pelo trocânter maior e pelo epicôndilo lateral do fêmur; lateralmente sobre a perna, na linha formada pelo epicôndilo lateral do fêmur e pelo maléolo lateral da fíbula; e no dorso do pé. A coleta e o tratamento inicial dos dados foram realizados no software Motive (Tracker 2.0.2) e consistia na captura dos movimentos, nomeação dos pontos e cálculos das coordenadas de quadril, coxa, perna e pé com 6 graus de liberdade (Translação X, Y e Z e rotações de Euller YXZ). Foram extraídas para análise as variáveis: ângulo de flexão/extensão do quadril e joelho no momento do contato do pé com o solo; ângulo de dorsiflexão/flexão plantar do tornozelo no momento do contato do pé com o solo; ângulo de adução/abdução do quadril no momento do contato do pé com o solo; máxima adução do quadril durante o apoio; ângulo de inversão/eversão do tornozelo no momento do contato do pé com o solo; máxima eversão do tornozelo durante o apoio; e máxima flexão do joelho durante o apoio. Também foram analisadas as variáveis cinemáticas espaço-temporais: frequência de passada, comprimento de passada e distância do pé à projeção do quadril no momento do contato do pé com o solo. 
A sessão experimental era iniciada com um breve aquecimento na própria esteira, efetivado por meio de 2 minutos na intensidade de $70 \%$ da velocidade estabelecida para o teste de corrida. Após o aquecimento, o teste de corrida foi realizado durante 5 minutos, na velocidade estabelecida para cada voluntário. Os parâmetros cinéticos e cinemáticos foram coletados durante os últimos 20 segundos, do $5^{\circ}$ minuto. Além dos parâmetros biomecânicos, foi avaliada a PSE.

\section{Tratamento dos dados}

Todos os equipamentos foram conectados a uma placa de aquisição analógica NI USB6210 (National Instruments, EUA) para sincronização. Todos os dados foram tratados e analisados no software MatLab R2015a (Mathworks, EUA). Os dados de FRS foram filtrados utilizando um filtro passa-baixa recursivo do tipo Butterworth de quarta ordem, com frequência de corte de $60 \mathrm{~Hz}$ e normalizados pelo peso corporal. Os dados cinemáticos foram filtrados utilizando um filtro passa-baixa recursivo do tipo Butterworth de segunda ordem, com frequência de corte de $10 \mathrm{~Hz}$.

\section{ANÁLISE ESTATÍSTICA}

Os resultados são apresentados com média e desvio padrão. Para comparação entre grupos foi realizado o Teste t de Student. A normalidade e homocedasticidade dos dados foram verificadas pelos testes de Kolmogorov-Smirnov e Levene respectivamente. O nível de significância adotado foi de 5\%. Todas as análises foram realizadas no software SigmaStat 3.5.

\section{RESULTADOS}

A velocidade média utilizada na corrida em esteira para os grupos GMaF e GMeF foi de $8,6 \pm 1,0 \mathrm{~km} / \mathrm{h}$ e 9,5 $\pm 1,5 \mathrm{~km} / \mathrm{h}$ respectivamente $(p=0,182)$, com PSE 2,3 $\pm 1,0$ e 2,9 \pm 0,4 respectivamente $(p=0,128)$. Não foram identificadas diferenças significativas entre os grupos para as variáveis da componente vertical da FRS (tabela 1), cinemáticas espaçotemporais (tabela 1), e cinemáticas das articulações do quadril, joelho e tornozelo (tabela 2). 
Tabela 1 - Variáveis da componente vertical da força de reação do solo e variáveis cinemáticas espaço-temporais durante a corrida. Onde: impT - impulso total; Fy 1 - $1^{\circ}$ pico de força; tFy 1 tempo para o $1^{\circ}$ pico de força; TDF - taxa de desenvolvimento de força; freq - frequência de passada; comp - comprimento de passada; dist_pé_quad - distância do pé à projeção do quadril no momento do contato com o solo.

\begin{tabular}{lccc}
\hline & GMaF & GMeF & p \\
\hline impT (pc.s) & $0,367 \pm 0,021$ & $0,368 \pm 0,025$ & 0,974 \\
Fy1 (pc) & $1,77 \pm 0,30$ & $1,97 \pm 0,47$ & 0,350 \\
tFy1 (s) & $0,074 \pm 0,017$ & $0,070 \pm 0,022$ & 0,777 \\
TDF (pc/s) & $67,41 \pm 25,79$ & $79,70 \pm 24,54$ & 0,345 \\
& & & \\
freq (passada/s) & $1,331 \pm 0,085$ & $1,335 \pm 0,089$ & 0,915 \\
comp (m) & $1,801 \pm 0,190$ & $1,968 \pm 0,262$ & 0,167 \\
dist_pé_quad (m) & $0,328 \pm 0,034$ & $0,313 \pm 0,045$ & 0,467 \\
\hline
\end{tabular}

Fonte: construção dos autores.

Tabela 2 - Variáveis cinemáticas das articulações do quadril, joelho e tornozelo durante a corrida. Onde: quad_fe_c - ângulo de flexão/extensão do quadril no momento do contato; quad_aa_c - ângulo de adução/abdução do quadril no momento do contato; máx_adu_quad_a - máxima adução do quadril durante apoio; joel_fe_c - ângulo de flexão/extensão do joelho no momento do contato; máx_flex_joel_a - máxima flexão do joelho durante apoio; torn_df_c - ângulo de dorsiflexão/flexão plantar do tornozelo no momento do contato; torn_ie_c ângulo de inversão/eversão do tornozelo no momento do contato; máx_eve_torn_a - máxima eversão do tornozelo durante apoio. Para quadril e joelho, valores positivos indicam flexão e adução e valores negativos indicam extensão e abdução. Para o tornozelo, valores positivos indicam dorsiflexão e inversão e valores negativos indicam flexão plantar e eversão.

\begin{tabular}{lccc}
\hline & GMaF & GMeF & P \\
\hline quad_fe_c $\left(^{\circ}\right)$ & $23,5 \pm 4,3$ & $25,8 \pm 3,9$ & 0,303 \\
quad_aa_c $\left(^{\circ}\right)$ & $2,4 \pm 2,6$ & $1,8 \pm 5,0$ & 0,748 \\
máx_adu_quad_a $\left(^{\circ}\right)$ & $4,1 \pm 2,7$ & $4,8 \pm 3,7$ & 0,678 \\
& & & \\
joel_fe_c $\left(^{\circ}\right)$ & $3,8 \pm 5,4$ & $6,1 \pm 6,2$ & 0,488 \\
máx_flex_joel_a $\left(^{\circ}\right)$ & $29,2 \pm 3,6$ & $32,9 \pm 4,1$ & 0,085 \\
& & & \\
torn_df_c $\left(^{\circ}\right)$ & $9,3 \pm 4,4$ & $10,1 \pm 4,8$ & 0,710 \\
torn_ie_c $\left(^{\circ}\right)$ & $6,1 \pm 5,2$ & $2,7 \pm 1,3$ & 0,089 \\
max_eve_torn_a $\left(^{\circ}\right)$ & $-10,5 \pm 2,3$ & $-12,1 \pm 2,5$ & 0,209 \\
\hline
\end{tabular}

Fonte: construção dos autores 


\section{DISCUSSÃO}

Considerando que o treinamento de força é recomendado como uma estratégia tanto para prevenção de lesão, como para aumentar o desempenho de praticantes de corrida, o presente estudo teve como objetivo comparar a biomecânica da corrida entre indivíduos praticantes de musculação, com diferentes níveis de força absoluta, em 10RM, no exercício agachamento livre. Decidimos determinar a capacidade de produzir força de membros inferiores pelo agachamento livre, por se tratar de um exercício frequentemente utilizado para este propósito; por reconhecidamente solicitar os principais músculos utilizados durante a corrida; e por ser um exercício tradicional e largamente utilizado nos programas de treinamento de força (SCHOENFELD, 2010).

Para o protocolo de corrida em esteira, optamos por uma corrida em intensidade moderada, de maneira que os resultados obtidos pela PSE, aferidos a partir da escala de percepção subjetiva de esforço adaptada de Foster e colaboradores (2001), nos permite inferir que a intensidade desejada foi alcançada. Além disso, não houve diferença significativa na velocidade de corrida entre os grupos (GMaF 8,6 $\pm 1,0 \mathrm{~km} / \mathrm{h}$ e GMeF 9,5 $\pm 1,5 \mathrm{~km} / \mathrm{h}(p=0,182)$ ).

De maneira geral, os resultados obtidos nesse estudo mostraram não haver diferença na biomecânica da corrida, avaliada por meio de variáveis da componente vertical da FRS, cinemáticas espaço-temporais e cinemáticas das articulações de quadril, joelho e tornozelo, entre indivíduos com diferentes níveis de força absoluta de membros inferiores.

Avaliar parâmetros espaço-temporais durante a corrida se faz relevante pois mudanças observadas no comportamento dessas variáveis podem indicar a ocorrência de outras alterações biomecânicas que necessitam de uma análise mais detalhada. No entanto, nosso estudo não apontou diferenças no comprimento e frequência de passada entre os grupos GMaF e GMeF. De fato, estudo prévio mostrou que para corredores recreacionais de longa distância, a adição de um programa de treinamento de força, de alta intensidade para membros inferiores, durante 8 semanas, não foi capaz de promover alterações no comprimento e frequência de passada em corrida de intensidade moderada (FERRAUTI; BERGERMANN; FERNANDEZ-FERNANDEZ, 2010). No mesmo sentido, a adição de um programa de treinamento de força, por meio de exercícios pliométricos, também não foi capaz de promover alterações na frequência de passada em corredores treinados de meia e longa distâncias (SAUNDERS et al., 2006) e no comprimento e frequência de passada em corredores 
treinados de cross-country (PAAVOLAINEN et al., 1999). Por outro lado, no estudo de Hayes; Bowen e Davies (2004) foi observado uma relação negativa entre variação do comprimento de passada e força muscular, de maneira que os autores sugerem que a força de resistência de extensores de quadril (concêntrica e excêntrica) e de flexores de joelho (excêntrica) são importantes para manter estável o padrão da corrida até a exaustão.

Outra variável espaço-temporal avaliada em nosso estudo foi a distância do pé à projeção do quadril no momento do contato com o solo, a qual pode ser relacionada com maior frenagem no sentido ântero-posterior (HEIDERSCHEIT et al., 2011; LIEBERMAN et al., 2015). Entretanto, não observamos alterações para esta variável.

Tendo como premissa que grande parte das lesões provenientes da prática da corrida ocorrem pelo acúmulo de sobrecargas repetitivas nos tecidos musculoesqueléticos (MAAS et al., 2018), entende-se que é importante investigar as variáveis associadas com as forças que surgem na interação do aparelho locomotor com o solo, a FRS, que se equivale a somatória dos produtos da aceleração da massa de todos os segmentos do corpo, e é considerado um indicativo de sobrecarga mecânica (AMADIO; SERRÃO, 2007). Em relação ao impulso total, como não observamos diferença entre os grupos, podemos inferir que não ocorreram alterações na oscilação vertical do centro de massa. Outro aspecto importante para ser considerado é o comportamento em conjunto dos parâmetros relacionados à força de impacto na corrida, pois nos permite dimensionar de maneira mais adequada a sobrecarga mecânica imposta pelas forças externas. No entanto, não observamos diferenças entre os grupos nas variáveis analisadas (i.e. $1^{\circ}$ pico de força vertical, tempo para o $1^{\circ}$ pico de força vertical e taxa de desenvolvimento de força), o que nos permite assumir que a capacidade de atenuar a sobrecarga da força vertical durante a corrida é semelhante, independente da capacidade de produzir força absoluta de membros inferiores. Semelhante ao contexto de nosso achado, o estudo de Moffit e colaboradores (2020), realizado com universitários corredores de longa distância, não encontrou associação entre o $1^{\circ}$ pico de força vertical e a força muscular de extensores de joelho e quadril, medida por meio de 1RM no agachamento livre e por máxima contração isométrica em dinamômetro isocinético. Ainda, no estudo de Paavolainen e colaboradores (1999), a adição de um programa de treinamento de força, por meio de exercícios pliométricos, também não foi capaz de acarretar alteração no componente vertical da FRS em corredores treinados de cross-country. 
Como não encontramos diferenças significativas entre os grupos nas variáveis cinemáticas de quadril, joelho e tornozelo, os resultados destes dados reforçam nossa interpretação de que a capacidade de atenuar a sobrecarga mecânica durante a corrida não depende da capacidade de produzir força absoluta de membros inferiores. Como exemplo, levamos em consideração o comportamento cinemático da articulação do joelho, a qual está intimamente relacionada com a absorção de energia mecânica gerada na corrida (MILLER; BRENT EDWARDS; DELUZIO, 2015). No presente estudo, não observamos diferenças entre GMaF e GMeF nas angulações de flexão do joelho, tanto no momento do contato, quanto durante a fase de apoio na corrida. Por outro lado, em estudo com corredores universitários de longa distância, Moffit e colaboradores (2020) encontraram uma fraca associação entre maior capacidade de 1RM no agachamento, com maior pico de flexão, menor pico angular de rotação interna e menor pico de momento de rotação interna de joelho durante fase de apoio na corrida; de maneira que os autores sugerem que por além de ser relevante para o desempenho na corrida, a força muscular apresenta importante papel no aumento da proteção do aparelho locomotor.

Da mesma forma, não identificamos diferença entre grupos, na angulação de flexão do quadril no momento de contato do pé com o solo. Ademais, a articulação do quadril também é considerada de grande importância para a manutenção da integridade do aparelho locomotor, sobretudo em relação aos ângulos de adução durante a corrida. O aumento do ângulo de adução do quadril durante a corrida tem sido relacionado com maior risco de lesão, como é o caso da dor patelofemoral (NOEHREN et al., 2012; NOEHREN; HAMILL; DAVIS, 2013; WILLSON; DAVIS, 2008). O aumento da adução do quadril pode acarretar um alinhamento inadequado entre a patela e o fêmur, gerando maior compressão no compartimento lateral da patela (HUBERTI; HAYES, 1984; POWERS, 2010). Entretanto, não encontramos diferenças entre os grupos nos ângulos de adução do quadril, tanto no momento de contato, quanto durante a fase de apoio na corrida. Nossos achados corroboram com o estudo de Moffit e colaboradores (2020), o qual não encontraram associação entre capacidade de 1RM no agachamento, com maior adução de quadril na corrida. Em contraponto, no estudo de Snyder e colaboradores (2009), realizado com mulheres saudáveis; curiosamente e contrariando a hipótese dos autores, um programa de fortalecimento dos músculos abdutores e rotadores externos do quadril, acarretou maior adução de quadril na corrida. Ainda assim, presumindo que a fraqueza dos músculos abdutores de quadril pode estar relacionada com maior risco de 
lesão (BECKER; NAKAJIMA; WU, 2018), parece ser importante considerar o fortalecimento destes músculos como estratégia para reduzir a probabilidade de lesão.

Em relação à articulação do tornozelo, como não encontramos diferenças para os ângulos de dorsiflexão/flexão plantar e inversão/eversão no momento do contato, podemos assumir que a geometria de colocação do pé no solo foi a mesma entre os grupos. Outro aspecto importante sobre a articulação do tornozelo são os movimentos excessivos relacionados com a pronação e supinação, pois estão associados com maior risco à integridade do aparelho locomotor (BECKER; NAKAJIMA; WU, 2018; CLANSEY et al., 2012). No entanto, não verificamos diferenças entre os grupos nas angulações de inversão no contato; e máxima eversão durante a fase de apoio na corrida.

Em suma, nossos resultados mostraram não haver diferença na biomecânica da corrida entre indivíduos com diferentes níveis de força absoluta em 10RM, no exercício agachamento livre. Estes achados podem ser explicados pelo fato de que o exercício em questão é pouco específico para a corrida devido as características de contração muscular, de maneira que mais estudos, como por exemplo, utilizando exercícios com acúmulo e restituição de energia elástica, podem contribuir para melhor compreensão da relação entre força muscular e a mecânica da corrida.

\section{CONCLUSÕES}

Indivíduos treinados com diferentes níveis de força absoluta em 10RM, no exercício agachamento livre, não apresentaram diferenças na biomecânica da corrida. Tal fato não significa que algum nível de força não seja importante para o controle de sobrecarga mecânica na corrida, portanto, é aconselhável a realização de exercícios específicos de fortalecimento muscular para indivíduos que desejam utilizar a corrida como parte de seu programa de condicionamento físico ou como esporte.

\section{REFERÊNCIAS BIBLIOGRÁFICAS}

ADAMS, Douglas e colaboradores. Validity and reliability of a commercial fitness watch for measuring running dynamics. Journal of orthopaedic and sports physical therapy, v. 46, n. 6, p. 471-476, 2016.

ALCARAZ-IBAÑEZ, Manuel; RODRÍGUEZ-PÉREZ, Manuel. Effects of resistance training on performance in previously trained endurance runners: a systematic review. Journal of sports 
sciences, v. 36, n. 6, p. 613-629, 2018.

AMADIO, Antonio Carlos; SERRÃO, Júlio Cerca. Contextualização da biomecânica para a investigação do movimento: fundamentos, métodos e aplicações para análise da técnica esportiva. Revista brasileira de educação física e esporte, v. 21, p. 61-85, 2007.

BALSALOBRE-FERNÁNDEZ, Carlos; SANTOS-CONCEJERO, Jordan; GRIVAS, Gerasimos V. Effects of strength training on running economy in highly trained runners: a systematic review with meta-analysis of controlled trials. Journal of strength and conditioning research, v. $30, n .8$, p. 2361-2368, 2016.

BECKER, James; NAKAJIMA, Mimi; WU, Will F. W. Factors contributing to medial tibial stress syndrome in runners: a prospective study. Medicine and science in sports and exercise, $\mathrm{v}$. 50, n. 10, p. 2092-2100, 2018.

BLAGROVE, Richard C. e colaboradores. Strength and conditioning habits of competitive distance runners. Journal of strength and conditioning research, v. 34, n. 5, p. 1392-1399, 2020.

BOHM, Sebastian; MERSMANN, Falk; ARAMPATZIS, Adamantios. Human tendon adaptation in response to mechanical loading: a systematic review and meta-analysis of exercise intervention studies on healthy adults. Sports medicine - open, v. 1, n. 1, 2015.

BOREL, Wyngrid Porfírio e colaboradores. Prevalence of injuries in brazilian recreational street runners: meta-analysis. Revista brasileira de medicina do esporte, v. 25, n. 2, p. 161-167, 2019.

CLANSEY, Adam C. e colaboradores. Effects of fatigue on running mechanics associated with tibial stress fracture risk. Medicine and science in sports and exercise, v. 44, n. 10, p. 19171923, 2012.

COMFORT, Paul e colaboradores. Relationships between strength, sprint, and jump performance in well-trained youth soccer players. Journal of strength and conditioning research, v. 28, n. 1, p. 173-177, 2014.

COMFORT, Paul; BULLOCK, Nathan; PEARSON, Stephen J. A comparison of maximal squat strength and 5-, 10-, and 20-meter sprint times, in athletes and recreationally trained men. Journal of strength and conditioning research, v. 26, n. 4, p. 937-940, 2012.

DAVIS, Irene S.; BOWSER, Bradley J.; MULLINEAUX, David R. Greater vertical impact loading in female runners with medically diagnosed injuries: a prospective investigation. British journal of sports medicine, v. 50, n. 14, p. 887-892, 2016.

FERRAUTI, Alexander; BERGERMANN, Mathias; FERNANDEZ-FERNANDEZ, Jaime. Effects of a concurrent strength and endurance training on running performance and running economy in recreational marathon runners. Journal of strength and conditioning research, v. $24, n$. 10 , p. 2770-2778, 2010. 
FIELDS, Karl B. e colaboradores. Prevention of running injuries. Current sports medicine reports, v. 9, n. 3, p. 176-182, 2010.

FOSTER, Carl e colaboradores. A new approach to monitoring exercise training. Journal of strength and conditioning research, v. 15, n. 1, p. 109-115, fev. 2001.

HAYES, Philip R.; BOWEN, Sarah J.; DAVIES, Emma J. The relationships between local muscular endurance and kinematic changes during a run to exhaustion at vVO2max. The journal of strength and conditioning research, v. 18, n. 4, p. 898, 2004.

HEIDERSCHEIT, Bryan C. e colaboradores. Effects of step rate manipulation on joint mechanics during running. Medicine \& science in sports \& exercise, v. 43, n. 2, p. 296-302, 2011.

HUBERTI, Helmut H.; HAYES, Wilson C. Patellofemoral contact pressures. The influence of qangle and tendofemoral contact. The journal of bone and joint surgery. American volume, v. 66, n. 5, p. $715-724,1984$.

JOHNSTON, Christopher A. M. e colaboradores. Preventing running injuries. Practical approach for family doctors. Canadian family physician Medecin de famille canadien, v. 49, p. 1101 1109, 2003.

LAVCANSKA, Vesna; TAYLOR, Nicholas F.; SCHACHE, Anthony G. Familiarization to treadmill running in young unimpaired adults. Human movement science, v. 24, n. 4, p. 544-557, 2005.

$\mathrm{LI}$, Fei e colaboradores. Effects of complex training versus heavy resistance training on neuromuscular adaptation, running economy and 5-km performance in well-trained distance runners. PeerJ, v. 2019, n. 4, p. e6787, 2019.

LIEBERMAN, Daniel E. What we can learn about running from barefoot running: an evolutionary medical perspective. Exercise and sport sciences reviews, v. 40, n. 2, p. 63-72, 2012.

LIEBERMAN, Daniel E. e colaboradores. Effects of stride frequency and foot position at landing on braking force, hip torque, impact peak force and the metabolic cost of running in humans. The journal of experimental biology, p. 3406-3414, 2015.

MAAS, Ellen e colaboradores. Novice runners show greater changes in kinematics with fatigue compared with competitive runners. Sports biomechanics, v. 17, n. 3, p. 350-360, 2018.

MIKKOLA, Jussi e colaboradores. Effect of resistance training regimens on treadmill running and neuromuscular performance in recreational endurance runners. Journal of sports sciences, v. 29, n. 13, p. 1359-1371, 2011.

MILLER, Ross H.; BRENT EDWARDS, William; DELUZIO, Kevin. J. Energy expended and knee joint load accumulated when walking, running, or standing for the same amount of time. Gait and posture, v. 41, n. 1, p. 326-328, 2015. 
MOFFIT, Tyler J. e colaboradores. Association between knee-and hip-extensor strength and running-related injury biomechanics in collegiate distance runners. Journal of athletic training, v. 55, n. 12, p. 1262-1269, 2020.

MUCHA, Mattew D. e colaboradores. Hip abductor strength and lower extremity running related injury in distance runners: a systematic review. Journal of science and medicine in sport, v. 20, n. 4, p. 349-355, 2017.

NAWOCZENSKI, Deborah A.; LUDEWIG, Paula M. Electromyographic effects of foot orthotics on selected lower extremity muscles during running. Archives of physical medicine and rehabilitation, v. 80, n. 5, p. 540-544, 1999.

NOEHREN, Brian e colaboradores. Proximal and distal kinematics in female runners with patellofemoral pain. Clinical biomechanics, v. 27, n. 4, p. 366-371, maio 2012.

NOEHREN, Brian; HAMILL, Joseph; DAVIS, Irene. Prospective evidence for a hip etiology in patellofemoral pain. Medicine and science in sports and exercise, v. 45, n. 6, p. 1120-1124, 2013.

PAAVOLAINEN, Leena e colaboradores. Explosive-strength training improves $5-\mathrm{km}$ running time by improving running economy and muscle power. Journal of applied physiology, $v .86$, n. 5, p. 1527-1533, 1999.

POWERS, Christopher M. The influence of abnormal hip mechanics on knee injury: a biomechanical perspective. Journal of orthopaedic and sports physical therapy, v. 40, n. 2, p. 42-51, 2010.

QUEEN, Robin M.; GROSS, Michael T.; LIU, Hsin-Yi. Repeatability of lower extremity kinetics and kinematics for standardized and self-selected running speeds. Gait and posture, v. 23, n. 3, p. 282-287, 2006.

SAUNDERS, Philo U. e colaboradores. Short-term plyometric training improves running economy in highly trained middle and long distance runners. The journal of strength and conditioning research, v. 20, n. 4, p. 947-954, 2006.

SCHOENFELD, Brad J. Squatting kinematics and kinetics and their application to exercise performance. Journal of strength and conditioning research, v. 24, n. 12, p. 3497-3506, 2010.

SNYDER, Kelli R. e colaboradores. Resistance training is accompanied by increases in hip strength and changes in lower extremity biomechanics during running. Clinical biomechanics, v. 24, n. 1, p. 26-34, 2009.

VAN DER WORP, Maarten P. e colaboradores. Injuries in runners; a systematic review on risk factors and sex differences. Plos one, v. 10, n. 2, p. 1-18, 2015.

VAN GENT, R. N. e colaboradores. Incidence and determinants of lower extremity running injuries in long distance runners: a systematic review. British journal of sports medicine, $v$. 
41, n. 8, p. 469-480, 2007.

WILLSON, John D.; DAVIS, Irene S. Lower extremity mechanics of females with and without patellofemoral pain across activities with progressively greater task demands. Clinical biomechanics, v. 23, n. 2, p. 203-211, 2008.

WISLØFF, Ulrik e colaboradores. Strong correlation of maximal squat strength with sprint performance and vertical jump height in elite soccer players. British journal of sports medicine, v. 38, n. 3, p. 285-288, 2004.

YAMAMOTO, Linda M. e colaboradores. The effects of resistance training on endurance distance running performance among highly trained runners: a systematic review. Journal of strength and conditioning research, v. 22, n. 6, p. 2036-2044, 2008.

\section{Dados do primeiro autor:}

Email:wplima22@gmail.com

Endereço: Laboratório de Biomecânica da Escola de Educação Física e Esporte da Universidade de São Paulo, Rua Professor Mello Moraes, 65, Cidade Universitária, São Paulo, SP, CEP: 05508030, Brasil.

Recebido em: 29/06/2021

Aprovado em: 28/07/2021

\section{Como citar este artigo:}

LIMA, Wilson Pereira e colaboradores. Comparação biomecânica da corrida entre indivíduos com diferentes níveis de força de membros inferiores. Corpoconsciência, v. 25, n. 2, p. 252268, mai./ ago., 2021. 